\title{
Cryostructurization of polymeric systems for developing macroporous cryogel as a foundational framework in bioengineering applications
}

\author{
ANUJ TRIPATHI* (D) and JOSE SAVIO MELO \\ Nuclear Agriculture and Biotechnology Division, Bhabha Atomic Research Centre, \\ Mumbai 400 085, Maharashtra, India \\ E-mail: anujtri@barc.gov.in
}

MS received 2 April 2019; revised 6 June 2019; accepted 13 June 2019

\begin{abstract}
Among various fabrication techniques to produce a porous scaffold, thermally-induced phase separation at controlled cryogenic condition leads to the formation of a porous polymeric cryostructure alias cryogel or cryomatrix. Cryostructurization is one of the simple and versatile methods of synthesizing a highly porous and interconnected architecture. The process of cryostructurization is present in comparable fabrication approach of fabricating advanced porous biomaterials with precise control over multiple compositions of precursor units, spatial distributions for accomplishing effective recapitulation of mechanical properties and architectural accuracy at micron-scale with bioactive functionality. The cryogenically-structured polymeric scaffolds are of noteworthy fundamental and applied interest in multi-disciplinary areas of science and showed promising matrices in various biotechnological and biomedical areas. The large interconnected pores in cryogels open up a range of applications like the three-dimensional substrate for cell growth, bioreactor for continuous production of scarce molecules, bio-processing and protein purification, an adsorbent for environmental remediation, and biosensor fabrication. Over the past two decades, significant attention to these materials with rigorous expansion in their new designs, and remarkable growth in the number of the publications and patents on cryostructured porous materials has been noticed. This article is covering the progress in cryostructurization technology in the last few decades with principles underlying the mechanism of cryogelation, process optimization, and the recent trend in cryogel for biomedical and bioengineering applications.
\end{abstract}

Keywords. Cryostructurization; cryogelation; cryogel; porous-biomaterials; polymer scaffolds.

\section{Introduction}

One of the most stimulating and gratifying areas of advanced materials science research engrosses the applications of biomaterials to biotechnology and healthcare, especially to regenerative medicine. Since one decade, the development of advanced functional polymeric biomaterials and their application to biomedicine have dramatically improved due to their tremendously important functional features. In addition, various medical devices and diagnostic products that employ biomaterials are currently being used and are performing well in various clinical practices. The demand in the biomaterials' market is growing rapidly due to the advancement into instrumentation and miniaturization technologies. A recent press report released by Markets and Markets studies on the global biomaterials market has forecast the growth of biomaterials market which is poised to reach USD 149.17 billion by 2021 from USD 70.90 billion in 2016, at a CAGR of $16.0 \%$ from 2016 to 2021 . $^{1}$ According to the report, increasing disease incidence, higher demand for prosthetics and other implantable devices, technological advancements, and raising awareness on tissue engineering are the driving factors of biomaterials market growth. Despite biomedical applications, polymeric biomaterials have gained increasing interest in various bioengineering applications due to natural inheritance and acceptance, low toxicity, and simple disposal procedures. Biomaterials are being investigated to produce economically viable and disposable bioreactors for continuous production and separation

*For correspondence 

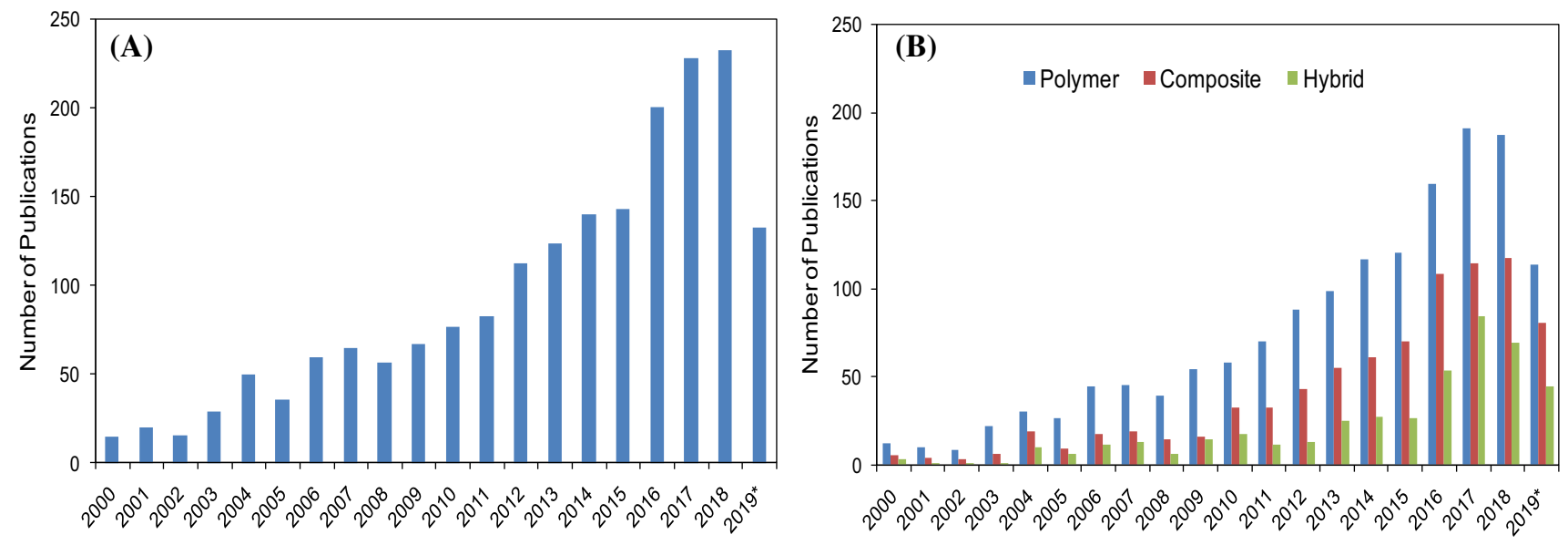

Figure 1. A trend in cryogel research in the past two decades (A) and publication based on its types i.e., polymeric cryogels, composite cryogels and hybrid cryogels (B). (*Data for the year 2019 is given as per the record of Science-Direct accessed on 27 March 2019).

of industrially important molecules, and also for the filtration matrices. With the advent of novel technologies and new application-oriented research, there has been a need for developing new interdisciplinary research on polymers, materials, and processes to put the novel knowledge into biological applications.

In biomedical applications, the classified types of biomaterials (like metallic, ceramic, polymers, natural) require special porous architecture with precise physicochemical features to integrate into the biological systems and also to support the biological function. However, the absence of interconnected porosity and elastic properties have undermined the potential utilization of hydrogel systems in many biotechnological and bioengineering applications. The interconnectivity between pores and homogeneous pore distribution in biomaterials plays a key role in their performances, especially for cell mobility for functional tissue reconstruction, high-throughput applications, mass flow, and bioreactors designing, etc. Therefore, a wide range of fabrication approaches has been exploited to generate the required porosity in the biomaterials. $^{2}$ Among many novel compositions and designing approaches of biomaterials, porous polymeric biomaterials is an important class which has attracted increasing interest as a carrier of chromatography, protein synthesis, drug delivery, tissue engineering and others due to their unique interconnected porous property. The introduction of the interconnected porous network in a polymer system can be governed by the use of a porogen material that either disrupts the polymer network or gets entrapped in the polymer system during the gel formation and is later removed without affecting the structural architecture. The cryostructuring approach of producing supermacroporous hydrogels at subzero temperature is known as cryogelation, and the produced gel is coined 'cryogel' or 'cryomatrix. ${ }^{3,4}$

Recent statistics show that the trend in cryogel research is paving the way for improving current practices in biomedical and bioengineering fields. There were around 2043 articles found on 'ScienceDirect' that included the keyword 'Cryogel,' out of that 1890 articles published from 2000 to 2019, which suggest that interest is increasing in cryogel research around the globe for advanced multidisciplinary applications (Figure 1a). Moreover, an increasing trend has also been monitored in the cryogel publications based on its composition, wherein polymeric cryogel has major contribution followed by its composite and hybrid preparations (Figure 1b).

Varieties of cryogel compositions have been fabricated and examined for various applications since it has been evolved as one of the potential porous material. At the beginning of cryogel research, it has been explored majorly for bioprocessing and environmental applications (like separation, purification, and remediation). However, in the last one decade it has shown potential applications in biomedical field majorly tissue-engineering, drug delivery and sensor applications (Figure 2).

\section{Thermally induced phase separation (TIPS)}

Thermally-induced phase separation (TIPS) is a process that involves the de-mixing of a polymer solution by changing the temperature. This induces the separation of polymer phase to the solvent phase, however, the rate of separation completely depends upon the cooling temperature, molecular weight and freezing point of polymer precursors and 


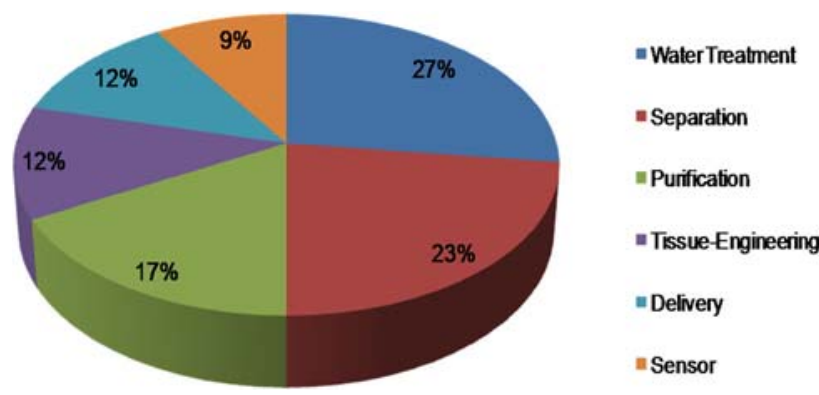

Figure 2. Major application areas and their per cent contribution in cryogel research in the last two decades.

viscosity of the solution. The greater leeway of this approach is to obtain well-interconnected pores within the polymer network. Interestingly, the porosity, pore diameter, and its size can be modulated during synthesis by changing the preparatory parameters, wherein temperature plays a critical role. For example, when a polymeric aqueous solution starts getting a temperature effect by changing its temperature from high to low, a process of nucleation occurs in the metastable region of a solution. If the process of nucleation is slow (i.e., system temperature is close to the solution freezing temperature), the growth of water crystals (porogen) will increase that result in large interconnected pores will be obtained in the scaffold. Besides, quick nucleation (i.e., a larger gap between freezing temperature of system and solution) causes small water crystals growth during the process of polymer phase separation and gelations resulting in small interconnected pores developments in the scaffold. By utilizing the advantage of TIPS, a gradient porosity can be achieved in a polymer system by treating it at gradient temperature, which is easy to tune and a single step process.

\section{Cryogel: the origin}

The meaning of words 'cryogels' or 'cryostructures' that include the syllable 'cryo' (from the Greek $\kappa \$ b 0 \varsigma$ (kryos) meaning frost) rather explains itself as the polymer gels and 3-D structures formed by means of the cryogenic process. In this context, cryogels are gel systems that develop in moderately frozen solutions of precursor's entities potentially capable of gelling by thermally induced phase separation. ${ }^{4}$

In a series of fabricating and inventing advanced three-dimensional porous polymeric systems with suitable applicability, cryostructurization has been evolved as a promising technology. In the early 1970s, it is known that cryogenic treatment (involves the process of freezing followed by frozen incubation and lastly thawing) of a concentrated aqueous solution of precursors produces a viscoelastic porous gel, so-called Cryogel. Although, freeze-thaw based gelation process was empirically observed long before. Cryogels gained importance in the early 1980s when Lozinsky and his co-workers at the Institute of Organo-Element Compounds, Russian Academy of Sciences (INEOS RAS), Moskva, Russia performed dedicated research on the process of cryogelation. ${ }^{5}$ Their initial studies were focused on understanding the mechanism of cryostructurization of polymeric systems to produce cryogel and characterizing the macroporous architecture of these cryogels for understanding the effect of crosslinking at sub-zero temperature, the effect of different solvent systems, and also the role of precursor concentration in obtaining a suitable porosity in the cryogel. Porous hydrogels can be classified on the basis of the following;

A) On the basis of appearance
1. Monoliths
2. Discs
3. Sheets
4. Beads/Granules

B) On the basis of preparation methods
1. Aerogel
2. Xerogel
3. Cryogel

C) On the basis of chemical structure
1. Polymeric
2. Hybrid
3. Composite

D) On the basis of polymer distribution in the gel
1. Mixed
2. Intercalated

E) On the basis of pore distribution

1. Microporous

2. Mesoporous

3. Macroporous

\section{Process of cryostructurization}

In the process of cryostructurization (Figure 3), the monomers, oligomers and/or polymer precursors are first dissolved in a suitable solvent such as water and dimethyl sulphoxide. The addition of crosslinker is case sensitive, depending on the requirement of interchain bonding within polymeric 3D network based on available reactive functional groups. Freezing of the dissolved precursor or colloidal dispersion below the crystallization point of solvent will form the solid crystals of solvent. The mechanism behind the formation of cryogels is thermally-induced phase 


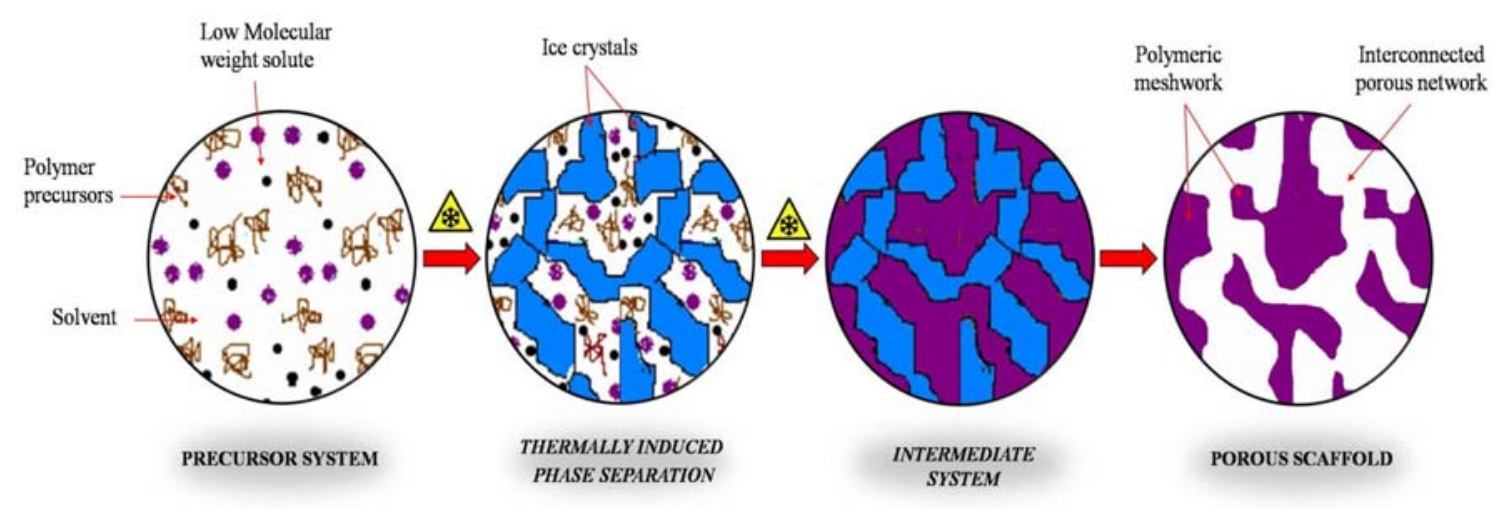

Figure 3. A schematic representation shows the process of cryostructurization of polymeric systems to produce porous cryogels after thawing of the frozen polymer system at room temperature.

separation (TIPS) that occurs when the solvent freezes below the crystallization point of the solvent and two phases are formed which are frozen solvent crystals and unfrozen liquid microphase (UFLMP). During the freezing period, solvent crystals keep growing around the UFLMP until they meet and form a continuous network of solvent crystals while polymerization reaction continues in UFLMP. These interconnected solvent crystals act as a porogen while the cryo-concentrated material in UFLMP results in the formation of pore wall in the cryogel. After the completion of the reaction, these gels are thawed at room temperature so that ice porogen will leave and form a web-like interconnected structure. One can find some similarity between cryogels and gels synthesized by freeze-drying. However, the principle difference in freeze-drying (lyophilization) and freeze-thawing (cryogelation) is the process of porogen elimination or dehydration of the polymer network. In freeze-drying, removal of the solvent is performed by the process of sublimation, wherein frozen solvent crystals are directly converted into gas at cryo-temperature $\left(\mathrm{T} \geq-50{ }^{\circ} \mathrm{C}\right)$ that leads to the formation of pores. In contrast, freeze-thawing involves the thawing of frozen crystals at room temperature $(\mathrm{T}=\mathrm{RT})$. However, a marked difference lies in the synthesis procedure and the gel formation does not take place due to the UFLM or the cryoconcentration but instead takes place normally like in other systems described elsewhere. ${ }^{6,7}$ Since a majority of their structure contains water or another solvent, the system still can be frozen and sublimed to form a porous structure. Unlike cryogelation, most lyophilization processes are completed over a period of desorption drying, which takes a long time and confers complications in the process of handling and energy consumption.

Cryogels form a highly porous interconnected architecture by the process of cryostructurization but their structural stability defined by the nature of interaction between the polymer chains. Therefore, types of crosslinking play an important role in stabilizing the porous cryostructures which can be a physical or a chemical cross-linking of polymer chains. Gelation under cryo-condition that involves physical-crosslinking via hydrogen bond formation between the hydroxyl and carboxyl moieties of polymer chains. ${ }^{8,9}$ It has been observed that physically crosslinked cryogels present increased physicochemical and mechanical stability. ${ }^{10,11}$ Physical crosslinking provides an advantage that it does not involve the use of unwanted toxic crosslinking agents which can affect later application if its residual impurities remain intact in the polymer network. Studies have been conducted on various polymers like agarose, alginate, polyvinyl alcohol (PVA), gelatin, and their composites like PVA-gelatin, alginate-gelatin. ${ }^{12-16}$ These cryostructures have majorly been explored in the immobilization and bioprocessing application. ${ }^{17}$

Besides this, chemically crosslinked cryostructures can be produced by chemical-induced crosslinking of functional groups and also lead a polymerization process. A common type of chemical crosslinking involves freeradical polymerization of monomers. However, proteins and polysaccharides get crosslinked via peptide bonding, Schiff base formation, Michael-addition reaction. These bonds have higher bond energy, and therefore, provide better stability compared to non-crosslinked and physically crosslinked polymer structures. ${ }^{18-20}$ Table 1 presents a list of crosslinked cryogels using different polymer precursors and mode of crosslinking.

\section{Morphology and other properties of cryogels}

Structurally, cryogel is a highly porous polymeric network having interconnected pores, which can be examined by different microscopic tools like light 


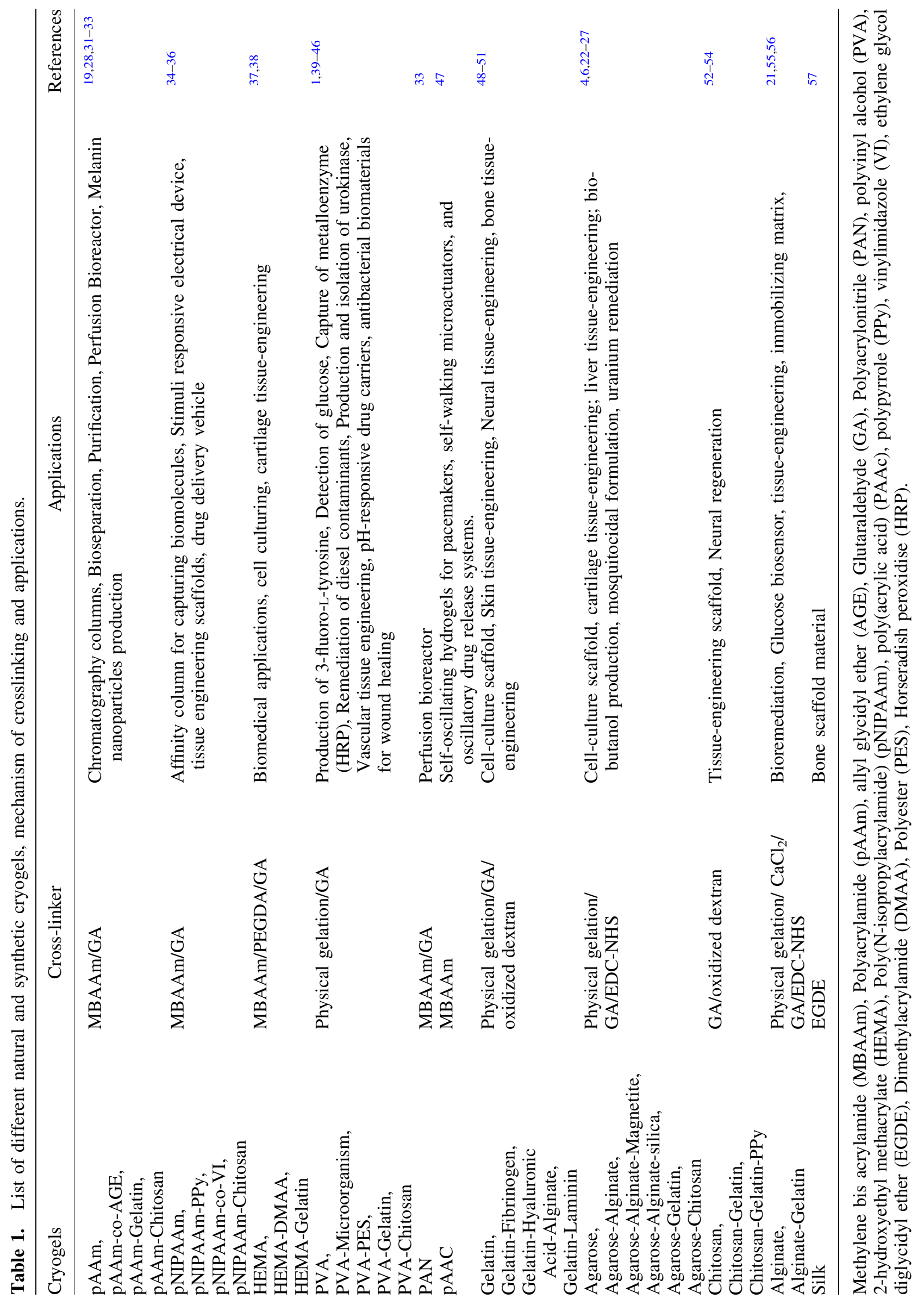


microscopy, scanning and transmission electron microscopy. In addition, the size of pores, its distribution, and total porosity of cryogel can be confirmed by mercury intrusion porosity analysis and microcomputed tomography. Our initial experiments support the authenticity of the principle of cryostructurization by monitoring the pores ranging from a few microns up to hundreds of microns and proved the heterogeneity in the cryostructures. ${ }^{4,6,21}$ The experimental details concluded with some interesting findings like an effect of the temperature regime during freezing and defreezing of the polymeric structure, the effect of precursor's concentration and type of crosslinker on crosslinking, and so on. ${ }^{21}$ According to the IUPAC nomenclature, the width of pores $<2 \mathrm{~nm}$ are classified as 'micropores,' and pores which are $>50 \mathrm{~nm}$ are defined as 'macroporous.' Considering this, the pores above this range are generally referred to as 'supermacroporous.' This is why, cryomatrices are sometimes also referred as supermacroporous polymeric systems, although, it is worth noting that the term 'supermacroporous' is not described officially in IUPAC.

Cryogel synthesis has been evolved over a period of time to produce them in various shapes and sizes. ${ }^{6}$
Production of a continuous structure of very large shape by this process is practically impossible and there is limited control on the morphology of the structure. The process of solvent removal from cryogels is much simpler and does not require lyophilization every time, which introduces further simplicity to the cryogelation system and imparts ease to the user during fabrication.

The physicochemical and mechanical properties of the cryogels are depending upon the polymer/precursor concentration, porosity and pores heterogeneity. In many applications where the flow of large mass is a desirable feature in a porous scaffold, the availability of large interconnected pores needs to be confirmed before application by performing simple swelling and permeability analysis. Several studies have shown quick swelling and high permeability in cryomatrices, ${ }^{6,22,23}$ which was found to be suitable for mammalian cell mobility and ingrowth for functional tissue genesis, ${ }^{4,24}$ controlled release, ${ }^{22}$ adsorption, ${ }^{23,25,26}$ solvent production, ${ }^{27}$ and separation applications. ${ }^{28}$ Additionally, cryogels have also been fabricated with variable viscoelastic properties and also low mechanical strength to high mechanical strength for a variety of applications. For example, addressing the cartilage
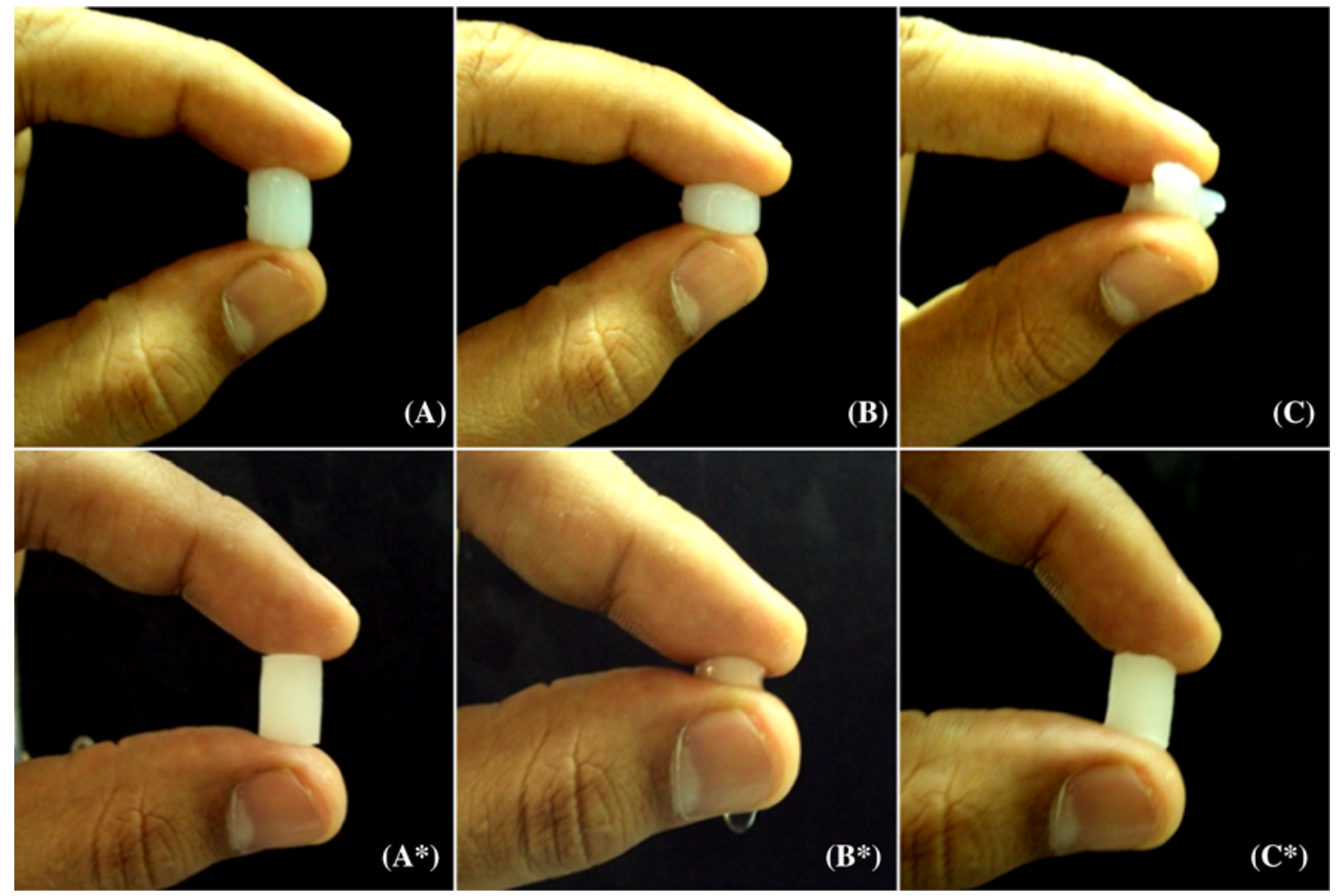

Figure 4. Elastic behavior of gels synthesized by cryostructurization i.e., cryogel (A), and room temperature synthesized hydrogel (A). Under stress, hydrogel compressed up to $50 \%$ (B) and above this, it showed permanent fracture (C). However, hydrated cryogel $\left(\mathrm{A}^{*}\right)$ compressed up to $80 \%$ of its original length $\left(\mathrm{B}^{*}\right)$ and returned to its original length upon relaxation $\left(C^{*}\right)$ shows its high elasticity. (Note: for the synthesis of gels all the parameters were remained constant except temperature, which was $-14{ }^{\circ} \mathrm{C}$ for cryogel and $25^{\circ} \mathrm{C}$ for hydrogel). 


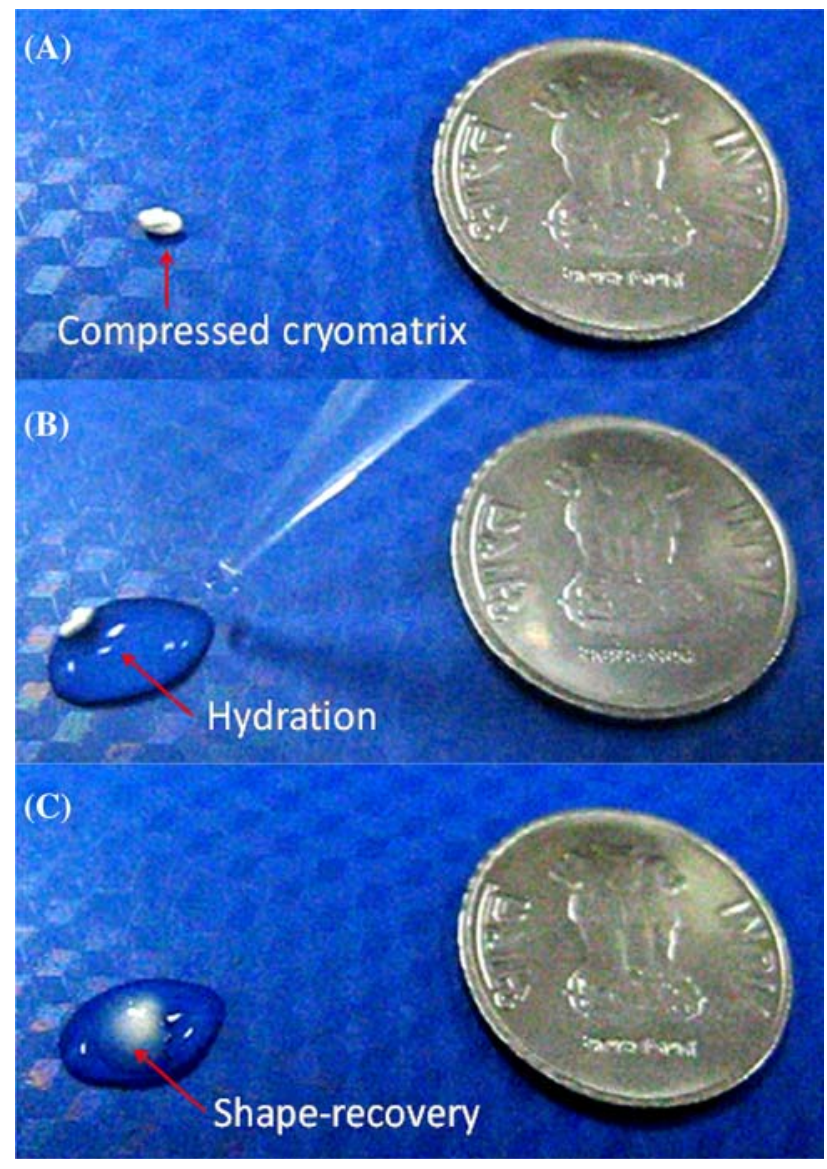

Figure 5. Shape-memory property of cryostructured porous polymeric matrix.

tissue regeneration and mimicking native cartilage structure-functions, gelatin-agarose cryogel with gradient porosity ${ }^{4}$ and gelatin-agarsoe-chitosan cryogel with high mechanical strength have been fabricated. It has been demonstrated that the mechanical property of cryogels can range from a few kilopascals (very soft cryomatrix) to several megapascals (very tough), which is depending on the type of application. ${ }^{4,6}$ One of the very simple ways to observe the elastic nature of hydrogel is to perform a manual stress-strain experiment, wherein the elastic cryogel remain unaffected upon compression but classical type of hydrogel deformed (Figure 4).

Additionally, inherited elastic nature of polymer along with cryostructurization process generates a shape-memory elastic cryogels (Figure 5). Besides these properties, surface topography is one of the important aspects to be precisely developed in any cryogel scaffolds for either the binding of a specific molecule or for the separation application. Such ligand-specific pore surfaces in cryogel have shown its potential thus explored by researchers as a versatile tool for translation application. There are several other features that can be introduced in the cryogel structures either before or after its synthesis. In conclusion, highly interconnected porous cryogel architectures have greater leeway to modulate its physicochemical and mechanical properties for the delivery of multi-functional scaffolds.

\section{Applications of cryogels}

The best-known information from the available published literature, the first few applications of cryogels were demonstrated in the field of immobilization of bacterial cells and enzymes onto the polymeric cryogel matrices. Now the advancement in the cryostructurization process has reached a new level. A recent study demonstrated the designing of a 3D porous bacterial cryo-sponge in a one-step process using single or multiple bacterial strains (Figure 6). This bacterial cryomatrix is presenting a novel approach of producing bioreactors with a high number of viable immobilised bacteria with a controlled 3D structure that has good mechanical stability, and cell viability that enables its reusable potential for various environmental, biotechnological, biological applications. ${ }^{29}$

Cryogels gained popularity in the 1990s when different groups around the world started exploiting the physicochemical properties of these supermacroporous materials for fundamental understanding in variety of applications. Since then, application-specific macroporous cryogels have been designed and applied extensively in various biotechnological and biomedical areas. Due to the simple fabrication procedure, unique and tunable physicochemical properties, large interconnected pores, use of aqueous non-toxic porogen, cryogels attract researchers from interdisciplinary fields. Because of this, cryogels were commercially developed for addressing bioseparation challenges. Cryogels have been successfully demonstrated its various applications. ${ }^{30,31}$ Our group is doing research on cryogels from more than a decade to explore the fundamental knowledge and translate it for sustainable applications. We have successfully developed various novel cryostructures using various synthetic and natural polymer procures and engineered them with suitable topography and design for improving the application potential. In particular, cryogels based on natural polymers such as alginate, collagen, gelatin, agarose, chitosan, etc., , 6, 21-28 have been investigated for various biological and biomedical applications because of their good biocompatibility, biodegradability, low toxicity, and other key advantages. Since the past one decade, this class of macroporous scaffolds have drawn great attention in the field of 

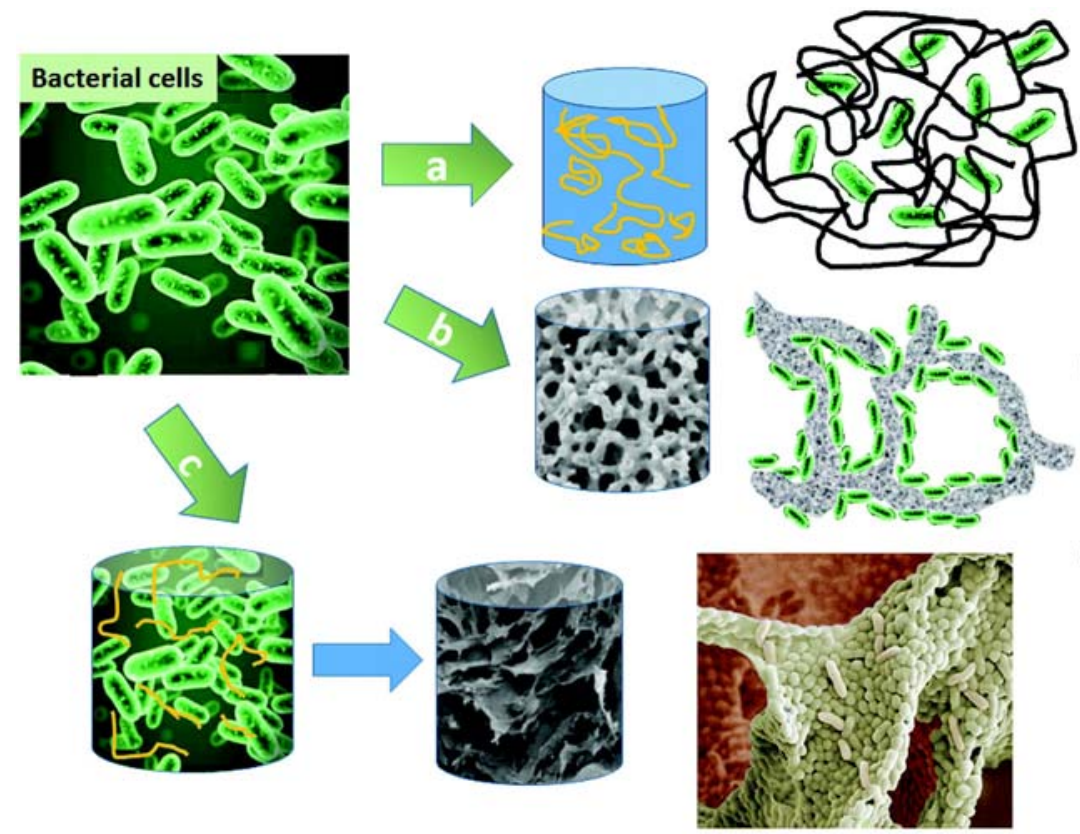

Immobilisation

(A) Entrapment into the bulk of the polymer (diffusion limitations, low number of cells with respect of polymer ratio)

(B) Formation of biofilm on the porous surface of the scaffold (long time for the biofilm formation)

(C) Cryogelation - direct cross-linking of the bacteria cells into biocryogel, bacterial sponge (one-step formation, high number of the cells with respect to polymer weight).

Figure 6. Schematic representation of bacterial cell immobilisation via (a) entrapment, (b) formation of biofilm on porous matrix, and (c) cryostructurization of bacterial cells to generate bacterial cryo-sponge, which shows their benefits and limitations. (Reproduced from RSC Adv. 2018830813 published by The Royal Society of Chemistry).

biomaterials for biomedical applications as an excellent substitute for 3D tissue constructs, wherein the growth of mammalian cells, either single types of cells or co-culturing of different cells have been demonstrated successfully (Figure 7).

Additionally, due to the shape-memory properties, some specific class of cryogels has the capacity to carry biofunctional molecules like cells and drugs with an injectable application for targeted delivery in regeneration therapy or immune-modulation. Several international research groups have successfully demonstrated the use of porous cryogels in the development of in vitro neo-tissues, bio-fuel production, bio-molecules imprinting and separation, remediation, floating mosquitocidal formulations, nanoparticles production, biosensors, etc. (Table 1), which presents them foundational framework as a new generation biomaterial in various biomedical and bioengineering applications.

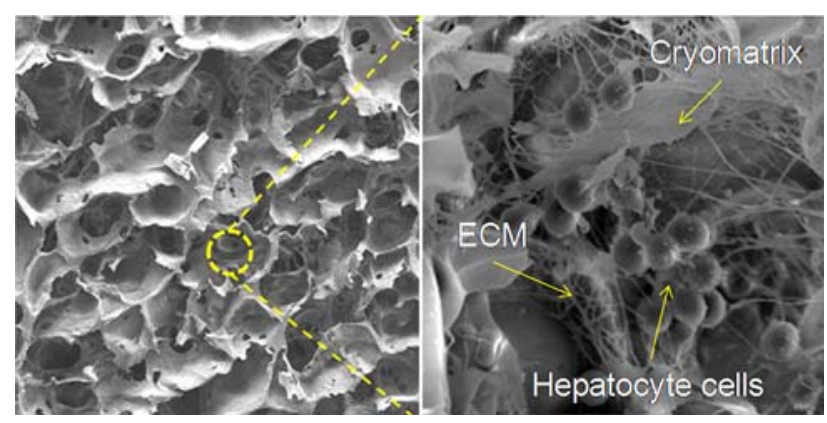

Figure 7. Cryomatrix used for the growth of hepatocyte cells for functional liver-like tissue functions.

\section{Conclusions}

Cryogels have been fabricated in various shapes, sizes with distinct physicochemical properties for specific applications ranging from separation, delivery, tissueengineering, sensors, immobilizing bioreactors etc., as discussed in this article. However, despite various technological advancements, a gap remains unfilled for broader translation of cryostructurized scaffolds for sustainable biological applications. For example, scaling-up of porous cryostructures without affecting their porous property is still a key area of research for advancing this field. Similarly, the effect of different three-dimensional microenvironment of pore-surfaces of the cryomatrices plays a crucial role in developing functional tissues and immunological understanding need to be addressed fundamentally for designing clinically-safe porous substrates. Research on interrelations between the structures of parent and resulting gels depending on regimes of the freeze-drying represents significant opportunities to be carried out as one of the future studies. Future work should demonstrate the potential of cryogel in scaling up the production process of industrially important molecules. Furthermore, advanced modification approaches can be introduced to tether the complex arrangement of multiple functional groups that can mimic the natural functionality for its use as a three-dimensional tissueengineered scaffolds. Such topographical modulation may closely mimic the tissue microenvironment and 
may assist in encouraging regeneration. Unlike the traditional designs of cryogels, its shape-memory property is advantageous and requires further exploration in biomedical applications. Cryostructurization of polymer systems has demonstrated ultimate use of cryostructures that are undoubtedly an advantageous scaffolding substrate for the use in a growing list of applications. After extensive scrutiny on its basic and applied behavior, cryostructures entail further consideration for its commercial delivery.

\section{Acknowledgement}

Authors would like to acknowledge the Department of Atomic Energy, Government of India, for financial assistance.

\section{References}

1. https://www.marketsandmarkets.com/PressReleases/ global-biomaterials.asp. Accessed on 31 March 2019

2. A Tripathi and J S Melo (Ed.) 2017 Advances in Biomaterials for Biomedical Applications (Singapore: Springer-Nature)

3. Kumar A and Tripathi A 2012 In Biotechnology in Biopolymers A Tiwari and R B Srivastava (Ed.) (United Kingdom: i-Smithers Repra Publication Ltd.) p. 233

4. Tripathi A, Kathuria N and Kumar A 2009 Elastic and macroporous agarose-gelatin cryogels with isotropic and anisotropic porosity for tissue engineering $J$. Biomed. Mater. Res. A 90680

5. Lozinsky V I, Galaev I Y, Plieva F M, Savina I N, Jungvid H and Mattiasson B 2003 Polymeric cryogels as promising materials of biotechnological interest Trends Biotechnol. 21445

6. Tripathi A and Kumar A 2011 Multi-featured macroporous agarose-alginate cryogel: synthesis and characterization for bioengineering applications Macromol. Biosci. 1122

7. Memic A, Colombani T, Eggermont L J, Rezaeeyazdi M, Steingold J, Rogers Z J, Navare K J, Mohammed H S and Bencherif S A 2019 Latest Advances in Cryogel Technology for Biomedical Applications Adv. Therap. 21800114

8. Lozinsky V I, Damshkaln L G, Brown R and Norton I T 2000 Study of cryostruc-turing of polymer systems. XIX. On the nature of intermolecular links in the cryogels of locust bean gum Polym. Int. 491434

9. Yokoyama F, Masada I, Shimamura K, Ikawa T and Monobe K 1986 Morphology and structure of highly elastic poly(vinyl alcohol) hydrogel prepared by repeated freez-ing-and-melting Colloid Polym. Sci. 264595

10. Eliasson A C and Kim H R 1992 Changes in rheological properties of hydroxypropyl potato starch pastes during freeze-thaw treatments i. a rheological approach for evalua-tion of freeze-thaw stability J. Texture Stud. 23279
11. Wu X Y, Huang S W, Zhang J T and Zhuo R X 2004 Preparation and character-ization of novel physically cross-linked hydrogels composed of poly(vinyl alcohol) and amine-terminated polyamidoamine dendrimer Macromol. Biosci. 471

12. Takamura A, Ishii F and Hidaka H 1992 Drug release from poly(vinyl alcohol) gel pre-pared by freeze-thaw procedure J. Control. Release 2021

13. Lozinsky V I 1998 Cryotropic gelation of poly(vinyl alcohol) solutions Russ. Chem. Rev. 67573

14. Lozinsky V Damshkaln L, Kurochkin I and Kurochkin I 2008 Study of cryostructur-ing of polymer systems: 28. Physicochemical properties and morphology of poly(vinyl alcohol) cryogels formed by multiple freezing-thawing Colloid J. 70189

15. El Fray M, Pilaszkiewicz A, Swieszkowski W and Kurzydlowski K J 2007 Morphology assessment of chemically modified cryostructured poly(vinyl alcohol) hydrogel Eur. Polym. J. 432035

16. Bajpai A and Saini R 2005 Preparation and characterization of biocompatible spongy cryogels of poly(vinyl alcohol)-gelatin and study of water sorption behaviour Polym. Int. 541233

17. Yu H, Xu X, Chen X, Lu T, Zhang P and Jing X 2007 Preparation and antibacte-rial effects of PVA-PVP hydrogels containing silver nanoparticles J. Appl. Polym. Sci. 103125

18. Sato T, Kita S and Otsu T 1979 Application of spin trapping technique to radical polym-erization, 18. Initiation mechanism of vinyl polymerization with $\mathrm{N}, \mathrm{N}$ dimethylaniline $N$-oxide/acid anhydride systems Makromol. Chem. 1801911

19. Plieva F M, Savina I N, Deraz S, Andersson J, Galaev I Y and Mattiasson B 2004 Characterization of supermacroporous monolithic polyacrylamide based matrices designed for chromatography of bioparticles $J$. Chromatogr. B $\mathbf{8 0 7} 129$

20. Savina I N, Cnudde V, D'hollander S, Van Hoorebeke L, Mattiasson B, Galaev I Y and Du Prez F 2007 Cryogels from poly (2-hydroxyethyl methacrylate): Macroporous, interconnected materials with potential as cell scaffolds Soft Matter 31176

21. Tripathi A, Vishnoi T, Singh D and Kumar A 2013 Modulated crosslinking of macroporous polymeric cryogel affect in-vitro cell adhesion and growth Macromol. Biosci. 13838

22. Tripathi A, Hadapad A B, Hire R S, Melo J S and D'Souza S F 2013 Polymeric macroporous formulations for the control release of mosquitocidal Bacillus sphaericus ISPC-8 Enzyme Microb. Technol. 53 398

23. Tripathi A, Melo J S and D'Souza S F 2013 Uranium (VI) recovery from aqueous medium using novel floating macroporous alginate-agarose-magnetite cryobeads J. Hazard. Mater. 246-247 87

24. Tripathi A and Melo J S 2015 Preparation of spongelike biocomposite agarose-chitosan scaffold with primary hepatocytes for establishing an in-vitro 3D liver tissue model RSC Adv. 530701

25. Tripathi A and Melo J S 2016 Synthesis of a lowdensity biopolymeric chitosan-agarose cryomatrix and its surface functionalization with bio-transformed 
melanin for the enhanced recovery of uranium (VI) from the aqueous sub-surfaces $R S C A d v$. 637067

26. Tripathi A and Melo J S 2019 Self-assembled biogenic melanin modulated surface-chemistry of biopolymerscolloidal silica composite porous matrix for the recovery of uranium Journal of Applied Polymer Science J. Appl. Poly. Sci. 13646937

27. Tripathi A, Sami H, Jain S R, Viloria-cols M, Zhuravleva N, Nilsson G, Jungvid H and Kumar A 2010 Improved bio-catalytic conversion by novel immobilization process using cryogel beads to increase solvent production Enzyme Microb. Technol. 4744

28. Tripathi A and Kumar A 2013 integrated approach for $\beta$-glucosidase purification from non-clarified crude homogenate using macroporous cryogel matrix Sep. Sci. Technol. 482410

29. Al-Jwaid A K, Berillo D, Savina I N, Cundy A B and Caplin J L 2008 One-step formation of three-dimensional macroporous bacterial sponges as a novel approach for the preparation of bioreactors for bioremediation and green treatment of water $R S C A d v .8$ 30813

30. Bakhshpour M, Idil N, Perçin I and Denizli A 2019 Biomedical applications of polymeric cryogels Appl. Sci. 9553

31. Saini A S, Tripathi A and Melo J S 2015 On-column enzymatic synthesis of melanin nanoparticles using cryogenic poly(AAM-co-AGE) monolith and its free radical scavenging and electro-catalytic properties $R S C$ Adv. 587206

32. Jain E and Kumar A 2013 Disposable polymeric cryogel bioreactor matrix for therapeutic protein production Nat. Protoc. 8821

33. Jain E and Kumar A 2009 Designing supermacroporous cryogels based on polyacry-lonitrile and a polyacrylamide-chitosan semi-interpenetrating network $J$. Biomater. Sci. Polym. Ed. 20877

34. Jain E, Damania A, Shakya A K, Kumar A and Sarin S K 2015 Fabrication of macroporous cryogels as potential hepatocyte carriers for bioartificial liver support Colloids Surf. B $\mathbf{1 3 6} 761$

35. Dainiak M B, Kumar A, Galaev I Y and Mattiasson B 2006 Detachment of affinity-captured bioparticles by elastic deformation of a macroporous hydrogel PNAS 103849

36. Deng Z, Guo Y, Ma P X and Guo B 2018 Rapid thermal responsive conductive hybrid cryogels with shape memory properties, photothermal properties and pressure-dependent conductivity J. Colloid Interface Sci. 526281

37. Savina I N, Cnudde V, D'Hollander S, Hoorebeke L V, Mattiasson B, Galaeva I Y and Prez F D 2007 Cryogels from poly(2-hydroxyethyl methacrylate): macroporous, interconnected materials with potential as cell scaffolds Soft Matter 31176

38. Singh D, Tripathi A, Nayak V and Kumar A 2011 Three-dimensional proliferation of chondrocytes on modelled elastic and macroporous HEMA-Gelatin cryogel J. Biomater. Sci. Polym. Ed. 221733

39. Lozinsky V I, Faleev N G, Zubov A L, Ruvinov S B, Antonova T V, Vainerman E S, Belikov V M and Rogozhin S V 1989 Use of PVA-cryogel entrapped citrobacter intermedius cells for continuous production of 3-fluoro-L-tyrosine Biotechnol. Lett. 1143

40. Doretti L, Ferrara D, Gattolin P, Lora S, Schiavon F and Veronese F M 1998 PEG-modified glucose oxidase immobilized on a PVA cryogel membrane for amperometric biosensor applications Talanta 45891

41. Cunningham C, Ivshina I, Lozinsky V, Kuyukina M and Philp J 2004 Bioremediation of diesel-contaminated soil by microorganisms immobilised in polyvinyl alcohol Int. Biodeterior. Biodegrad. 54167

42. Hajizadeh S, Kirsebom H, Leistner A and Mattiasson B 2012 Composite cryogel with immobilized concanavalin A for affinity chromatography of glycoproteins J. Sep. Sci. 352978

43. Kumar A, Bansal V, Nandakumar KS, Galaev IY, Roychoudhury P K, Holmdahl R and Mattiasson B 2006 Integrated bioprocess for the production and isolation of urokinase from animal cell culture using supermacroporous cryogel matrices Biotechnol. Bioeng. 93636

44. Vrana NE, Cahill P A and McGuinness G B 2010 Endothelialization of PVA/gelatin cryogels for vascular tissue engineering: effect of disturbed shear stress conditions J. Biomed. Mater. Res. A 941080

45. Pamfil D, Butnaru E and Vasile C 2016 Poly(vinyl alcohol)/chitosan cryogels as $\mathrm{pH}$ responsive ciprofloxacin carriers J. Polym. Res. 23146

46. Chhatri A, Bajpai J and Bajpai A K 2011 Designing polysaccharide-based antibacterial biomaterials for wound healing applications Biomatter 1189

47. Bilici C, Karayel S, Demir T T and Okay O 2010 Selfoscillating $\mathrm{pH}$-responsive cryogels as possible candidates of soft materials for generating mechanical energy J. Appl. Polym. Sci. 1182981

48. Singh D, Tripathi A, Zo S M, Singh D and HAN S S 2014 Synthesis of composite gelatin-hyaluronic acidalginate porous scaffold and evaluation for in-vitro stem cell growth and in-vivo tissue integration Colloids Surf. B 116502

49. Inci I, Kirsebom H, Galaev I Y, Mattiasson B and Piskin E 2013 Gelatin cryogels crosslinked with oxidized dextran and containing freshly formed hydroxyapatite as potential bone tissue-engineering scaffolds J. Tissue Eng. Regen. Med. 7584

50. Jurga M, Dainiak M B, Sarnowska A, Tripathi A, Plieva F M, Strojek L, Jungvid H, Kumar A, Firraz N and McGuckin C 2011 The performance of laminincontaining cryogel scaffolds in neural tissue regeneration Biomaterials 323423

51. Sarnowska A, Jablonska A, Jurga M, Dainiak M, Strojek L, Drela K, Wright K, Tripathi A, Kumar A, Jungvid H, Lukomska B, Forraz N, McGuckin C and DomanskaJanik K 2013 Encapsulation of mesenchymal stem cells by bio-scaffolds protects cells survival and attenuates neuroinflammatory reaction in injured brain tissue after transplantation Cell Transplant. 22 S67

52. Kathuria N, Tripathi A, Kar K K and Kumar A 2009 Synthesis and characterization of elastic and macroporous chitosan-gelatin cryogels for tissue engineering Acta Biomater. 5406

53. Berillo D, Elowsson L and Kirsebom H 2012 Oxidized dextran as crosslinker for chitosan cryogel scaffolds 
and formation of polyelectrolyte complexes between chitosan and gelatin Macromol. Biosci. 121090

54. Vishnoi T and Kumar A 2013 Comparative study of various delivery methods for the supply of alpha-ketoglutarate to the neural cells for tissue engineering Biomed. Res. Int. 2013294679

55. Fatoni A, Dwiasi D W and Hermawan D 2016 Alginate cryogel based glucose biosensor 2016 IOP Conf. Ser. Mater. Sci. Eng. 107012010
56. Shan L, Gao Y, Zhang Y, Yu W, Yang Y, Shen S, Zhang S, Zhu L, Xu L, Tian B and Yun J 2016 Fabrication and use of alginate-based cryogel delivery beads loaded with urea and phosphates as potential carriers for bioremediation Ind. Eng. Chem. Res. 55 7655

57. Ak F, Oztoprak Z, Karakutuk I and Okay O 2013 Macroporous silk fibroin cryogel Macromolecules 143 719 